\title{
A novel three-dimensional quantitative assessment method for abnormal root morphology of the maxillary premolars in vivo on cone-beam computed tomography
}

Jian Liu

Peking University School and Hospital of Stomatology

Xiao Xu

Peking University School and Hospital of Stomatology

Xian-E Wang

Peking University School and Hospital of Stomatology

Peng-Cheng Jia

Peking University School and Hospital of Stomatology

Meng-Qiao Pan

Peking University School and Hospital of Stomatology

Li Xu ( $\square$ xulihome@263.net)

Peking University School and Hospital of Stomatology

\section{Research Article}

Keywords: Cone-beam Computed Tomography, Root abnormality, Maxillary premolars, In vivo

Posted Date: March 3rd, 2022

DOI: https://doi.org/10.21203/rs.3.rs-1397878/v1

License: (c) (i) This work is licensed under a Creative Commons Attribution 4.0 International License.

Read Full License 


\section{Abstract \\ Background}

Previous studies have described and recorded abnormal root morphology. However, most of these studies were based on two-dimensional periapical or panoramic radiographs, and only a few studies have quantified it. We aimed to combine two-dimensional periapical radiographs and three-dimensional conebeam computed tomography (CBCT) to conduct qualitative judgment and quantitative analysis of normal and conical roots, and explore the clinical diagnostic method of normal and conical roots based on intraoral radiographs and CBCT.

\section{Methods}

The conical root was identified visually on periapical radiographs as the clinical gold standard. All teeth were divided into the cone-rooted teeth (CRT) or normal-rooted teeth (NRT) group. Furthermore, the differences in root length (RL), root surface area (RSA), and root volume (RV) of conical and normal roots in the maxillary premolars on CBCT were compared. Receiver operator characteristic curves were generated, and the areas the under curve (AUCs) and cutoff values were calculated to evaluate the diagnostic value for RV, RSA, RV/RL, and RSA/RL.

\section{Results}

The RSAs of NRT and CRT were $236.88 \pm 27.93 \mathrm{~mm}^{2}$ and $207.98 \pm 27.80 \mathrm{~mm}^{2}$, respectively $(P<0.05)$. The mean RV in the CRT group was lower than that in the NRT group, and the difference was statistically significant $\left(253.40 \pm 41.98 \mathrm{~mm}^{3}\right.$ vs. $\left.316.93 \pm 49.89 \mathrm{~mm}^{3}\right)$. The AUC values of the RSA/RL and RV/RL were 0.87 and 0.89 , respectively, and the best cutoff values were 19.61 for RSA/RL (if RSA/RL was < 19.61 , the teeth were considered CRT) and 24.05 for $R V / R L$ (if $R V / R L$ was $<24.05$, the teeth were considered CRT).

\section{Conclusions}

$\mathrm{CBCT}$ had a significant diagnostic value in the clinical evaluation of conical roots. RSA/RL and RV/RL were the best parameters with the largest AUC and high sensitivity and specificity.

\section{Background}

The root of the tooth is a decisive factor for the function, stability, and long-term retention of the tooth. Abnormal root morphology is commonly observed in clinics, including curved, slender, short, and conical roots [1-3]. It is seldom considered an extremely important factor in the treatment of periodontal 
diseases. Periodontitis is a chronic infectious disease with plaque biofilm as the initial factor, although its occurrence, progression, clinical severity, and prognosis are affected by many factors, such as age, smoking, local factors (such as dental anatomy and restoration, calculus), and systemic diseases [4-7]. Among the local susceptibility factors of the host, abnormal root morphology is of concern, especially in Chinese patients with aggressive periodontitis, who show root abnormality (RA) more commonly than do patients with chronic periodontitis or gingivitis $[3,8,9]$. Lü et al. proved that abnormal root morphology is an adverse prognostic factor in patients with aggressive periodontitis [8]. Abnormal root morphology often accelerates the progression of periodontitis, seriously affects the prognosis of periodontitis, and even leads to the failure of teeth and dentition to function properly. McGuire et al. showed that a poor crown-root ratio and poor root morphology were associated with tooth loss [10].

Previous studies have described and recorded abnormal root morphology [2, 9, 11-13]. However, most of these studies were based on two-dimensional periapical or panoramic radiographs, and only a few studies have quantified it. Lind first described the short-rooted anomaly, which was observed when the crown-root ratio was greater than 1.1 [14]. However, as a common type of RA, the conical root has not yet been clearly defined.

Therefore, this study aimed to combine two-dimensional periapical radiographs and three-dimensional (3D) cone-beam computed tomography (CBCT) to conduct qualitative judgment and quantitative analysis of normal and conical roots. The qualitative judgment of the conical root on periapical radiographs was referred to as a clinical diagnostic criterion. This study compared the differences in root length (RL), root surface area (RSA), and root volume (RV) of conical and normal roots in the maxillary premolars on $\mathrm{CBCT}$ and explored the clinical diagnostic method of normal and conical roots based on intraoral radiographs and CBCT.

\section{Methods}

\section{Teeth selection}

This study was approved by the Peking University Ethics Committee and Competent Authority (No. PKUSSIRB-202168154).

Periapical radiographs and $\mathrm{CBCT}$ results of the upper premolar were collected from the previous periodontitis case database of [blinded for review] for analysis. Intraoral periapical radiographs were obtained using the bisecting-angle projection technique. Scans of premolars in vivo were obtained using a CBCT scanner (NewTom VG, QR s.r.I.) at $110 \mathrm{kV}$ and $5 \mathrm{~mA}$. The field of view was $12 \mathrm{~cm} \times 8 \mathrm{~cm}$, and the layer thickness was $0.3 \mathrm{~mm}$.

The inclusion criteria were as follows: patients (1) aged 18-40 years with systemic health data, (2) with > 20 remaining teeth, (3) with clear periapical radiographs and CBCT of the upper premolar, and (4) with single and intact roots of the upper premolar. 
The exclusion criteria were as follows: patients (1) who were systemically unhealthy with tumour or metabolic disease, (2) with oral and maxillofacial acute inflammation or tumour, (3) with orthodontics history, (4) with root resorption, filling in the root, or root fracture of the premolar, (5) with crowding or malposition in the premolar region, (6) with curved-rooted, syncretic-rooted, or short-rooted teeth, and (7) with no periodontal ligament on radiographs.

\section{Conical root inspection}

The conical root was identified visually on periapical radiographs by three experienced periodontists. All teeth were divided into two groups, which were referred to as cone-rooted teeth (CRT) and normal-rooted teeth (NRT) groups.

\section{Measurements on periapical radiographs}

\section{Root width parameters}

The parameters of the upper premolars measured on periapical radiographs included the following: parameters of root width (PRW): the median points of the lines joining the mesial or distal cementoenamel junction (CEJ) point and apex were referred to as $M$ or $D$. The intersection point of the line $M D$ and the mesial or distal margin of the root was defined as M1 or D1: root width parameter = (M1D1-MD)/2.

\section{Measurements on cone-beam computed tomography}

\section{Root length}

Mimics (Quotation Mimics 21.0, Materialise Dental) was used for the analysis. After 3D reconstruction, the images were oriented in three perpendicular planes to measure the $\mathrm{RL}$ on the buccal-lingual plane. $\mathrm{RL}$ was defined as the distance between the apex and median point of the line that joins the buccal and lingual CEJ points.

\section{Root surface area and root volume}

After 3D reconstruction, the margin of the premolar was determined manually from every two layers on the cross-sectional and sagittal plane images. The crown and root portions were segmented separately by CEJ manually from each layer on the sagittal plane images. After segmentation, crown, root, and tooth masks were exported in STereoLithography format and parameters such as the RSA and RV were measured using Mimics (Figure 1). The RSA was calculated using the formula RSA $=\left(\mathrm{S}_{\mathrm{MT}}+\mathrm{S}_{\mathrm{MR}^{-}}\right.$ $\mathrm{S}_{\mathrm{MC}}$ /2 [15]. The RV can be calculated automatically using software. RSA/RL and RV/RL were calculated manually.

\section{Raw colour map}


All 3D original surface models from the same teeth (upper first premolar or upper second premolar) of different patients were unified in a common coordinate system and loaded in Geomagic (Geomagic Wrap 2017, 3D System). The average 3D surface models, which were the average of original models of same teeth, were created by achieving the position of the minimum distance of the corresponding marker points through coordinate transformation under the principle of least square method. Furthermore, 3D differences between normal and conical root teeth were calculated to assess the morphological differences.

To help visualise the differences, a 3D, colour-coded map for the differences between the normal and conical root teeth models was generated, where the normal root teeth were displayed as smaller (negative, blue), the same (0 surface distances, green), or larger (positive, red) than the conical root teeth. The blue to red colour-coded scale was standardised, allowing a proper comparison, and pure blue and red were set at $-1.145 \mathrm{~mm}$ and $1.145 \mathrm{~mm}$, respectively.

The raw colour map of the average models for NRT and CRT showed regions of statistically significant surface-to-surface differences (Figure 3).

\section{Sample size}

Based on the following formula, the sample size of this study was calculated:

$$
\begin{gathered}
n_{1}: n_{2}=1: k \\
n_{1}=\frac{k+1}{k}\left[\frac{\left(z_{\alpha / 2}+z_{\beta}\right) \sigma}{\delta}\right]^{2} \\
n_{2}=k n_{1}
\end{gathered}
$$

Based on the results of the preliminary trial, if $k a$, and $\beta$ values were $0.75,0.05$, and 0.20 , respectively, 54 teeth and 41 teeth were required for the NRT and CRT groups, respectively.

\section{Statistical analyses}

All statistical analyses were performed using Statistical Package for the Social Sciences Statistics version 25 software (IBM Corp., Armonk, NY, USA). Every measurement of the periapical radiograph and CBCT scan was repeated after 1 week by the same researcher, and the intra-examiner error was tested using a paired $t$ test. The intraclass correlation coefficient (ICC) was used to evaluate the magnitude of the measurement error. The mean of the two measurements was used in this study. The group $t$-test and nonparametric test were used for statistical analysis. $P<0.05$ was considered significant for all tests.

The RA diagnosed visually on periapical radiographs was referred to as the clinical gold standard for comparison, and the diagnostic criteria remained stable in the study. Receiver operator characteristic (ROC) curves were generated, and the area under curves (AUCs) were calculated to evaluate the 
diagnostic value for PRW, RV, RSA, RV/RL, and RSA/RL. After performing statistical analyses, the cutoff values were calculated for PRW, RV, RSA, RV/RL, and RSA/RL. The accuracy of the CBCT method in detecting root abnormalities was re-evaluated using the cutoff values. Subsequently, the sensitivity (Se), specificity (Sp), positive and negative predictive values, Youden index ( $\mathrm{YI})$, and positive and negative likelihood ratios were calculated.

\section{Results}

In total, 44 maxillary first premolars and 51 maxillary second premolars were enrolled in this study. The ICC values for all parameters were greater than 0.8 , indicating that the measurements had excellent reliability.

There was no statistical difference for the RL between NRT and CRT $(11.62 \pm 1.19 \mathrm{~mm}$ vs. $11.65 \pm 1.54 \mathrm{~mm}$, $P>0.05)$. The mean PRWs for the NRT and CRT groups were $0.48 \pm 0.12 \mathrm{~mm}$ and $0.33 \pm 0.16 \mathrm{~mm}$, respectively, and the difference was statistically significant. The mean RSAs for the NRT and CRT groups were $236.88 \pm 27.93 \mathrm{~mm}^{2}$ and $207.98 \pm 27.80 \mathrm{~mm}^{2}$, respectively $(P<0.05)$. The mean RV in the CRT group was lower than that in the NRT group, and the difference was statistically significant $\left(253.40 \pm 41.98 \mathrm{~mm}^{3}\right.$ vs. $316.93 \pm 49.89 \mathrm{~mm}^{3}$ ). The results shown in Table 1 indicated that PRW, RSA, and RV could be used in the clinical judgment of NRT and CRT.

Table 1. Comparison between normal-rooted and cone-rooted teeth based on RL, PRW, RSA, RV, RSA/RL, and RV/RL

\begin{tabular}{|lllllll|}
\hline & RL & PRW & RSA & RV & RSA/RL & RV/RL \\
\hline NRT & $11.62 \pm 1.19$ & $0.48 \pm 0.12$ & $236.88 \pm 27.93$ & $316.93 \pm 49.89$ & $20.42 \pm 1.86$ & $27.34 \pm 3.87$ \\
CRT & $11.65 \pm 1.54$ & $0.33 \pm 0.16$ & $207.98 \pm 27.80$ & $253.40 \pm 41.98$ & $17.88 \pm 1.31$ & $21.76 \pm 2.43$ \\
$P$ & 0.91 & $<0.01$ & $<0.01$ & $<0.01$ & $<0.01$ & $<0.01$ \\
\hline
\end{tabular}

Note: Data are presented as the mean \pm standard deviation.

Abbreviations: RL, root length, PRW, parameters of root width, RSA, root surface area, RV, root volume, NRT, normal root teeth, CRT, conical root teeth

The ROC curves of PRW, RSA, RV, RSA/RL, and RV/RL were determined (Figure 2). The AUC values of RSA/RL and RV/RL were 0.87 and 0.89 , respectively, and the best cutoff values were 19.61 for RSA/RL (if the RSA/RL was < 19.61, the tooth was considered a conical root) and 24.05 for RV/RL (if the RV/RL was $<24.05$, the tooth was considered a conical root) (Table 2 ). The results indicated that RSA/RL and RV/RL are excellent parameters for detecting normal and conical roots. CBCT results and clinical diagnoses were reanalysed using $2 \times 2$ contingency tables. Based on Table 3 , the accuracy of CBCT in detecting CRT was reevaluated using the cutoff value. The Se and YI of the RSA were lower than those of other 
parameters, and the $\mathrm{Yl}$ of the RV/RL was higher than that of the other parameters. RV/RL would be the most efficient parameter for the clinical detection of CRT based on the best critical point.

Table 2. Summary of AUCs and cutoff values for PRW, RSA, RV, RSA/RL, and RV/RL

\begin{tabular}{|llllll|}
\hline & PRW & RSA & RV & RSA/RL & RV/RL \\
\hline AUC & 0.80 & 0.76 & 0.84 & 0.87 & 0.89 \\
\hline Cutoff & 0.37 & 208.44 & 295.20 & 19.61 & 24.05 \\
\hline$P$ & 0.000 & 0.000 & 0.000 & 0.000 & 0.000 \\
\hline
\end{tabular}

Abbreviations: AUC, area under the curve, PRW, parameters of root width, RSA, root surface area, RV, root volume, $\mathrm{RL}$, root length

Table 3. Accuracy of PRW, RSA, RV, RSA/RL, and RV/RL for detecting normal-rooted and cone-rooted teeth

\begin{tabular}{|llllll|}
\hline & PRW & RSA & RV & RSA/RL & RV/RL \\
\hline Se & 0.73 & 0.51 & 0.83 & 0.95 & 0.88 \\
\hline Sp & 0.85 & 0.89 & 0.72 & 0.72 & 0.81 \\
\hline PV+ & 0.79 & 0.78 & 0.69 & 0.72 & 0.78 \\
\hline PV- & 0.81 & 0.71 & 0.85 & 0.95 & 0.90 \\
\hline YI & 0.58 & 0.40 & 0.55 & 0.67 & 0.69 \\
\hline LR+ & 4.94 & 4.61 & 2.99 & 3.42 & 4.74 \\
\hline LR- & 0.31 & 0.55 & 0.24 & 0.07 & 0.15 \\
\hline
\end{tabular}

Abbreviations: PRW, parameters of root width, RSA, root surface area, RV, root volume, RL, root length, Se, sensitivity, Sp, specificity, PV+, positive predictive value, PV-, negative predictive value, YI, Youden index, $\mathrm{LR}+$, positive likelihood ratio, LR-, negative likelihood ratio

The raw colour map of the average models for NRT and CRT showed regions of statistically significant surface-to-surface differences (Figure 3). As can be observed from the map, the red regions focused on the buccal and lingual root surfaces, and the yellow regions focused on the interproximal root surface. The results suggested that the overall dimension of the conical root was smaller than that of the normal root and not limited to the mesial-distal diameter.

\section{Discussion}

Root morphological abnormalities in teeth are common [1-3,16]. The initial root form is a key factor associated with the prognosis of periodontitis and tooth loss $[8,17]$. Among the local susceptibility 
factors of the host, root morphology is of concern, especially in Chinese patients with aggressive periodontitis, who show RA more commonly than do patients with chronic periodontitis or gingivitis $[3,8,9,18]$. The conical root is a typical type of root morphological abnormality that differs from the normal root in shape and dimensions. As can be observed from the results, the RSA and RV of the conical root were lower than those of the normal root without the influence of the RL, which suggests that the volume and surface area of the periodontal ligament of the conical root are smaller than those of the normal root. The geometric shape of the conical root is different from that of the normal root. Compared with the thick and long normal root, the conical root could bear a lower occlusal force and is more likely to exhibit tooth mobility. After periodontal destruction occurs, the normal occlusal force is significantly heavy to be borne by CRT, which may accelerate the progression of periodontitis.

Some studies show that atypical root shape, such as long, narrow, and deviated roots, increases the risk of apical root resorption during orthodontic treatment [2,19]. All of these studies are based on periapical radiographs. However, other studies have an inconsistent conclusion, and they hypothesised that abnormal root shape is not associated with root resorption [20].

Xu et al. showed that in periapical radiographs, there was a significant difference between conical and normal roots in terms of root width and quantitatively described the morphological difference between conical and normal roots in two-dimensional images for the first time [3]. There are few clinical studies related to conical roots, and they are mainly based on two-dimensional radiographs, such as periapical and panoramic radiographs $[2,12,13]$. This method is subject to certain bias due to the angle, position, and exposure of the X-ray projection and has significant limitations because it cannot reconstruct the 3D structure of the root and evaluate 3D data, such as the RSA and RV.

With the wide application of high-precision CBCT in clinics, 3D reconstruction techniques based on CBCT have increasingly developed, which makes it possible to measure the 3D characterisation of teeth in vivo. A previous study has focused on root resorption evaluation in patients with skeletal class III malocclusion and suggested that volume measurement based on CBCT provided a new sensitive method to detect root resorption [21]. The method has gradually become an important research method of root morphology, and the accuracy and reproducibility of the measurement of RSA and RV were high in a prior study [1]. Jia et al. showed that 3D reconstruction using CBCT was accurate and reliable for measuring the RSA [15]. Wang et al. showed that the in vivo measurement of tooth volume by CBCT was as accurate as in vitro micro-computed tomography measurement [22].

In this study, the RV and RSA of the conical root were lower than those of the normal root, which proved that the conical root differs with the normal root in morphology based on quantitative analysis. These results are similar to those of other studies. Ahlbrecht et al. evaluated the root morphology of maxillary incisors using CBCT and proved that 3D surface model construction for upper incisors is reproducible and 3D shape analysis using CBCT images allows a phenotypic characterisation of incisor root morphology, such as conical root, which refers to the root narrower in circumference, but may or may not have increased length relative to the overall sample average [1]. This result is similar to that of this study. 
As can be observed from the raw colour map, the conical root was narrower in the circumference than was the normal root, which may have influenced the occlusal stress distribution in the periodontal ligament, center of rotation, and center of resistance. This hypothesis will be proven by finite element analysis in the future.

\section{Conclusions}

This study showed that the CBCT method had a great diagnostic value in the clinical evaluation of conical roots of premolars. RSA/RL and RV/RL were the best parameters with the largest AUC $(>0.85)$ and high Se and $\mathrm{Sp}(\mathrm{YI}>0.65)$. The best cutoff values for RSA/RL and RV/RL were 19.61 and 24.05, respectively. Finally, more clinical studies are needed to develop a CBCT method for detecting conical roots of the anterior teeth.

\section{Abbreviations}

3D three-dimensional

AUC area under the curve

CBCT cone-beam computed tomography

CEJ cemento-enamel junction

CRT cone-rooted teeth

ICC intraclass correlation coefficient

NRT normal-rooted teeth

PRW parameters of root width

$\mathrm{RL}$ root length

ROC receiver operator characteristic curve

RSA root surface area

$\mathrm{RV}$ root volume

Se sensitivity

Sp specificity

YI Youden index 


\section{Declarations}

\section{Ethics approval and consent to participate}

This study was approved by the Peking University Ethics Committee and Competent Authority (No. PKUSSIRB-202168154). Consent was obtained from all participants in this study. This study was performed in accordance with the 1964 Declaration of Helsinki and its later amendments or comparable ethical standards.

\section{Consent for publication}

Not applicable

\section{Availability of data and materials}

The datasets used and/or analysed during the current study are available from the corresponding author on reasonable request. The datasets generated and/or analysed during the current study are not publicly available due patient imaging data is a matter of personal privacy.

\section{Competing interests}

The authors declare that they have no competing interests.

\section{Funding}

This study was supported by the Beijing Municipal Science and Technology Commission grant (No. Z181100001718111).

\section{Authors' contributions}

Jian Liu and Xiao Xu completed data measurement and wrote the main manuscript text.

Xian-E Wang , Peng-Cheng Jia, Meng-Qiao Pan collected data.

Li Xu directed the whole work.

\section{Acknowledgements}

We would like to thank Editage (www.editage.cn) for English language editing.

\section{References}

1. Ahlbrecht CA, Ruellas ACO, Paniagua B, Schilling JA, McNamara JA, Cevidanes LHS. Threedimensional characterization of root morphology for maxillary incisors. PLOS ONE. 2017,12:e0178728. 
2. Mirabella AD, Artun J. Risk factors for apical root resorption of maxillary anterior teeth in adult orthodontic patients. Am J Orthod Dentofacial Orthop. 1995,108:48-55.

3. Xu L, Meng HX, Tian Y, Zhang L, Feng XH, Zhang G. [Evaluation of root abnormity in patients with aggressive periodontitis]. Zhonghua Kou Qiang Yi Xue Za Zhi. 2009,44:266-9.

4. Caton JG, Armitage G, Berglundh T, Chapple ILC, Jepsen S, Kornman KS, et al. A new classification scheme for periodontal and peri-implant diseases and conditions - Introduction and key changes from the 1999 classification. J Periodontol. 2018,89:S1-8.

5. Caton JG, Armitage G, Berglundh T, Chapple ILC, Jepsen S, Kornman KS, et al. A new classification scheme for periodontal and peri-implant diseases and conditions - Introduction and key changes from the 1999 classification. J Clin Periodontol. 2018,45:S1-8.

6. Tonetti MS, Greenwell H, Kornman KS. Staging and grading of periodontitis: Framework and proposal of a new classification and case definition. J Periodontol. 2018,89,Suppl 1:S159-72.

7. Tonetti MS, Greenwell H, Kornman KS. Staging and grading of periodontitis: Framework and proposal of a new classification and case definition. J Clin Periodontol. 2018,45,Suppl 20:S149-61.

8. Lü D, Meng H, Xu L, Wang X, Zhang L, Tian Y. Root abnormalities and nonsurgical management of generalized aggressive periodontitis. J Oral Sci. 2017,59:103-10.

9. Meng H, Xu L, Li Q, Han J, Zhao Y. Determinants of host susceptibility in aggressive periodontitis. Periodontol 2000. 2007,43:133-59.

10. McGuire MK, Nunn ME. Prognosis versus actual outcome. Il. The effectiveness of clinical parameters in developing an accurate prognosis. J Periodontol. 1996,67:658-65.

11. Levander $E$, Malmgren O. Evaluation of the risk of root resorption during orthodontic treatment: $A$ study of upper incisors. Eur J Orthod. 1988,10:30-8.

12. Ahuja PD, Mhaske SP, Mishra G, Bhardwaj A, Dwivedi R, Mangalekar SB. Assessment of root resorption and root shape by periapical and panoramic radiographs: A comparative study. J Contemp Dent Pract. 2017,18:479-83.

13. Sameshima GT, Asgarifar KO. Assessment of root resorption and root shape: Periapical vs panoramic films. Angle Orthod. 2001,71:185-9.

14. Lind V. Short root anomaly. Scand J Dent Res. 1972,80:85-93.

15. Jia P, Yang G, Hu W, Chung KH, Zhao Y, Liu M, et al. Comparison of in situ cone beam computed tomography scan data with ex vivo optical scan data in the measurement of root surface area. Oral Surg Oral Med Oral Pathol Oral Radiol. 2019,128:552-7.

16. Mcnamara CM, Garvey MT, Winter GB. Root abnormalities, talon cusps, dentes invaginati with reduced alveolar bone levels: Case report. Int J Paediatr Dent. 1998,8:41-5.

17. Mcguire MK, Nunn ME. Prognosis versus actual outcome. III. The effectiveness of clinical parameters in accurately predicting tooth survival. J Periodontol. 1996,67:666-74.

18. Liu J, Wang XE, Lv D, Qiao M, Zhang L, Meng HX, et al. [Association between root abnormalities and related pathogenic genes in patients with generalized aggressive periodontitis]. Beijing Da Xue Xue 
Bao Yi Xue Ban. 2020,53:16-23.

19. Sameshima GT, Sinclair PM. Predicting and preventing root resorption: Part I. Diagnostic factors. Am J Orthod Dentofacial Orthop. 2001,119:505-10.

20. Maués CP, Do Nascimento RR, Vilella Ode v. Severe root resorption resulting from orthodontic treatment: Prevalence and risk factors. Dent Press J Orthod. 2015,20:52-8.

21. Lu H, Gao J, Ma H, Li X. [Three-dimensional root resorption evaluation of maxillary anterior teeth in skeletal class III patients: A cone beam computed tomography study]. Zhonghua Kou Qiang Zheng Ji Xue Zhi. 2020,27:129-33.

22. Wang Y, He S, Yu L, Li J, Chen S. Accuracy of volumetric measurement of teeth in vivo based on cone beam computer tomography. Orthod Craniofac Res. 2011,14:206-12.

\section{Figures}
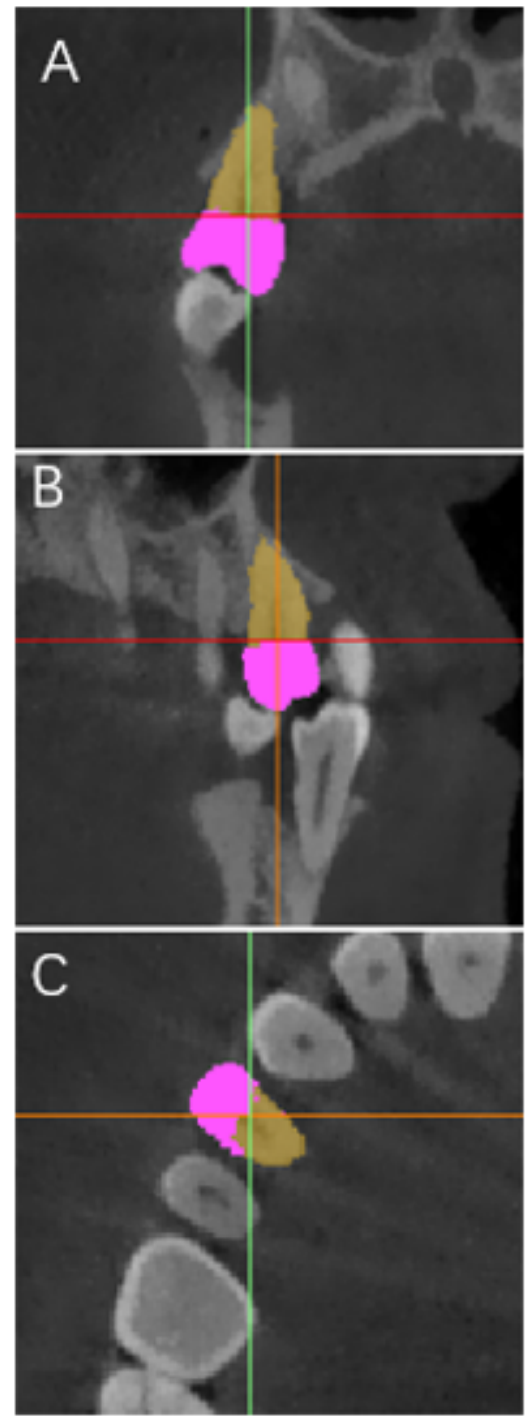

\section{Calculated Part}
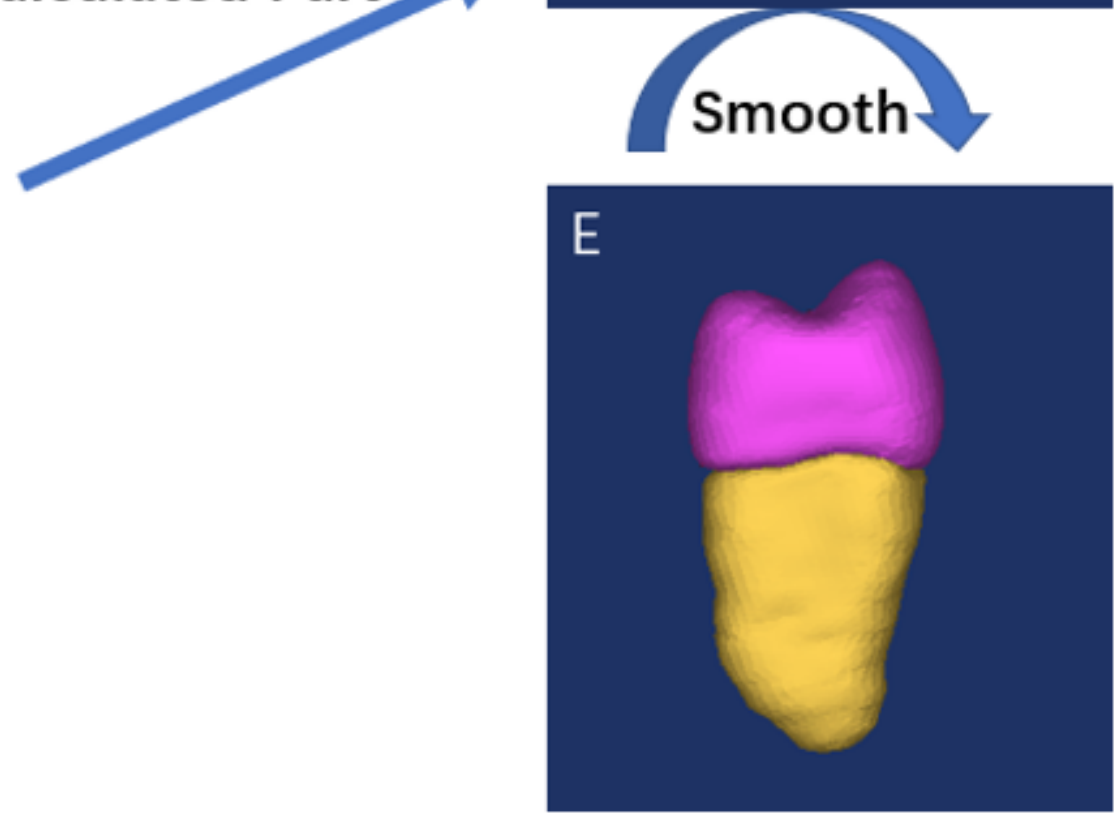
Figure 1

Schematic diagram of segmentation for crown, root, and tooth masks
A: Coronal plane
B: Sagittal plane
C:Axial plane
D: Initial three-dimensional image of tooth
E: Smoothened three-dimensional tooth model

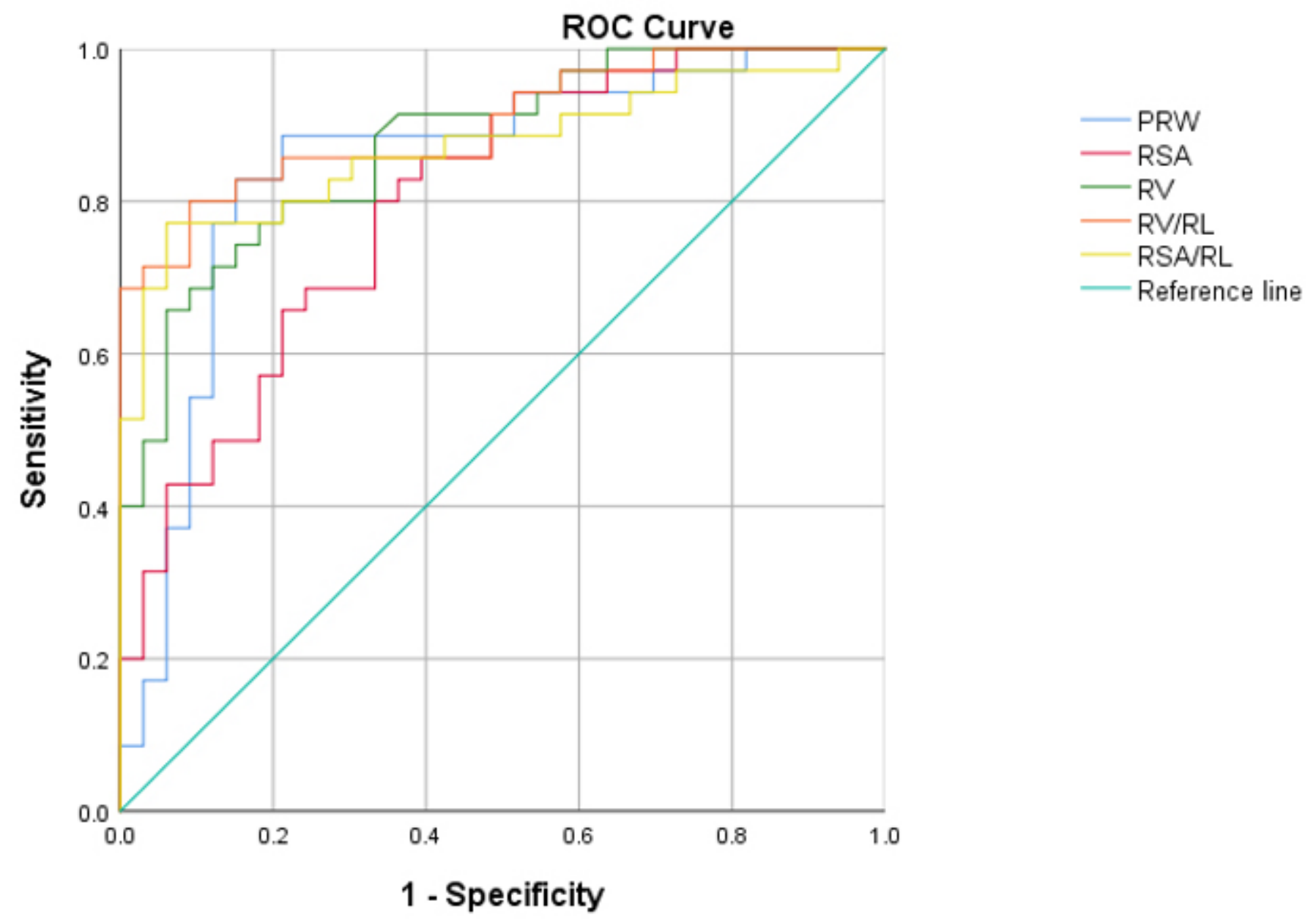

Figure 2

Receiver operator characteristic curves for root width, volume, surface area, and volume/length

PRW, parameters of root width, RL, root length, ROC, receiver operator characteristic curve, RSA, root surface area, RV, root volume 


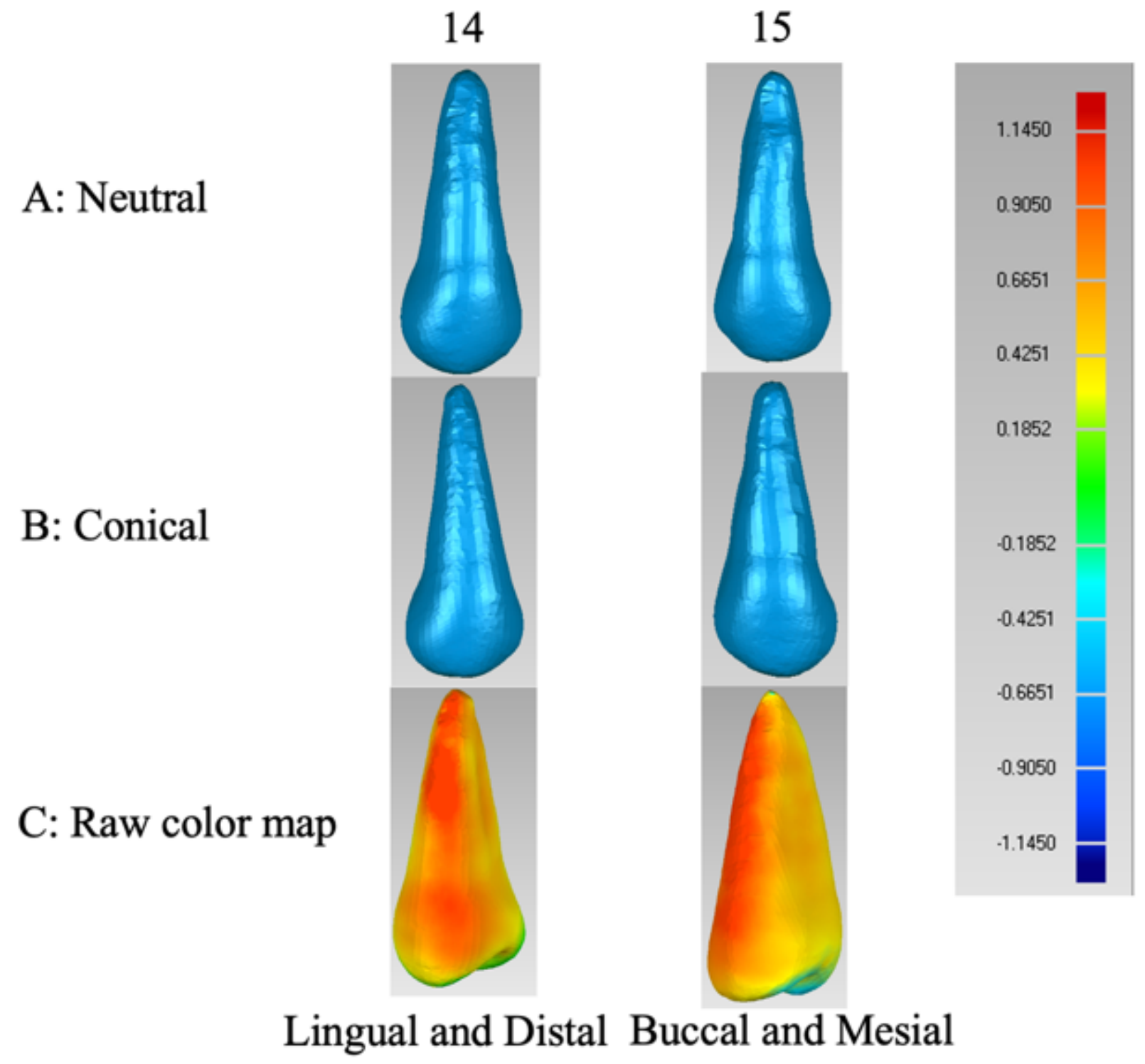

\section{Figure 3}

Raw colour map of the maxillary first and second premolars

A: Average models for the neutral maxillary premolars, B: average models for the conical maxillary premolars, C: raw colour map showing the regions of statistically significant surface-to-surface differences (cone as a reference) 